\title{
Lower Extremity Weakness in an Adolescent Female - A Rare Presentation of Ewing Sarcoma
}

\author{
Luke Addesso, BS, ${ }^{1}$ David Koenig, BS, ${ }^{2}$ Nadia Huq, MD $^{3}$ \\ ${ }^{1}$ Medical College of Wisconsin, Milwaukee, WI ${ }^{2}$ Des Moines University College of Osteopathic Medicine, Des Moines, IA ${ }^{3}$ Department of \\ Internal Medicine, Aurora Health Care, Milwaukee, WI
}

Background: Ewing sarcoma, a rare cause of cord compression, is predominantly of osseous origin but can also originate in soft tissues. Soft-tissue manifestations account for $<15 \%$ of all Ewing sarcoma tumors, and even fewer cases of Ewing sarcoma originating in the epidural space have been documented.

Case Report: A 19-year-old female presented to the emergency department for worsening low-back pain during the previous 6 months and numbness and weakness in her legs during the prior 2 weeks. Magnetic resonance imaging revealed an epidural mass at the L4-L5 level. Intravenous steroids were started for a presumed diagnosis of lymphoma. Orthopedic surgery consultants deferred computed tomography-guided biopsy of the mass out of concern for tumor seeding. Compression symptoms worsened to include foot drop and saddle anesthesia, prompting urgent radiation therapy. After the patient showed poor response to appropriate treatment for lymphoma, other malignant and infectious causes were considered. Biopsy was performed on day 3 of the patient's hospital stay, and by day 7, preliminary cytology results revealed Ewing sarcoma. Subsequent laminectomy and tumor resection produced immediate relief of pain, along with a gradual return of strength and sensation. The mass was found to be of soft-tissue origin and was classified as an extraosseous Ewing sarcoma. The patient was referred to a pediatric oncologist to complete the appropriate chemotherapy after diagnosis.

Conclusion: This case demonstrates how an uncommon manifestation of a rare disease can mimic a classic presentation of cord compression. Our aim is to bring awareness to this disease and to emphasize the importance of timely biopsy of any mass.

Keywords: Polyradiculopathy, sarcoma-Ewing, spinal cord compression

Address correspondence to Nadia Huq, MD, Department of Internal Medicine, Aurora Health Care, 1020 N. 12th St., Milwaukee, WI 53233. Tel: (763) 528-1653. Email: nadia.huq@aurora.org

\section{INTRODUCTION}

Ewing sarcoma, a rare malignancy primarily originating from bone, is most commonly found in children. Originally thought to be a distinct entity, Ewing sarcoma is now considered to be part of a larger group of malignancies called the Ewing sarcoma family of tumors that includes Ewing sarcoma, extraosseous Ewing sarcoma, primitive neuroectodermal tumor, and Askin tumor. ${ }^{1}$ These tumors are all thought to originate from a common mesenchymal progenitor cell and share a common chromosomal translocation of $\mathrm{t}(11 ; 22)$ (q24; q12), termed the EWSR1 translocation. ${ }^{1,2}$

Extraosseous Ewing sarcoma was first described in 1969, 48 years after the original discovery of Ewing sarcoma. ${ }^{3}$ Tefft et al established the foundational characteristics of extraosseous Ewing sarcoma as a soft-tissue tumor that lacks intraosseous destruction and exhibits a histologic pattern consistent with Ewing sarcoma. ${ }^{3}$ Soft-tissue manifestations account for $<15 \%$ of all Ewing sarcoma tumors. ${ }^{1}$ Compared to Ewing sarcoma, extraosseous Ewing sarcoma is more commonly found in females ( $46.6 \%$ vs $36.7 \%$ ), has a later age of onset (19.5 years vs 16.3 years), and preferentially arises in axial regions $(72.9 \%$ vs $54.2 \%) .{ }^{4} \mathrm{~A}$ study examining the distribution of 130 cases of extraosseous Ewing sarcoma reported that the most common locations of extraosseous Ewing sarcoma were the trunk (32\%), extremities (26\%), and head/neck (18\%). ${ }^{5}$ However, few cases of extraosseous Ewing sarcoma originating in the epidural space, as in our case, have been reported..$^{6-8}$ It is important to characterize epidural extraosseous Ewing sarcoma tumors to determine the prognostic significance and treatment implications.

We present the case of an adolescent female with 6 months of lower back pain and rapidly progressing neurologic findings who was incidentally found to have an extraosseous Ewing sarcoma of the lumbar epidural space.

\section{CASE REPORT}

A 19-year-old Caucasian female with no significant medical history presented to the emergency department (ED) with the complaint of progressively worsening lower back pain. Her initial back pain had occurred 6 months prior, a 
few days before her annual visit with her primary care provider (PCP). At the time of her annual examination, x-rays of the lumbar and thoracic spine were unremarkable. The patient completed a course of physical therapy without much improvement of her symptoms. The next evaluation of her back pain occurred 6 months later at an urgent care visit. The patient presented after a night out dancing that had exacerbated her back pain. Her legs suddenly began to feel numb and gave out, causing her to collapse. The patient was given a dose of dexamethasone and was started on $40 \mathrm{mg}$ prednisone daily, but the medications did not provide relief. The patient followed up with her PCP the next morning, who recommended that she immediately present to the ED for imaging.

On presentation to the ED, the patient had lumbar back pain radiating down both legs with associated numbness and weakness in her bilateral legs and feet. She reported no interim trauma or injury. Physical examination revealed findings concerning for spinal cord compression, including decreased strength in both lower extremities, decreased right patellar reflex, and absent Achilles reflexes bilaterally. Routine laboratory workup was unremarkable. Magnetic resonance imaging (MRI) of the lumbar spine revealed a dorsal epidural and posterior paraspinal mass at L4-L5 that severely narrowed the thecal sac and right neural foramen (Figure 1). On the night of admission, consultants from orthopedic spinal surgery and neuro-oncology agreed that the initial concern was for lymphoma, based on the imaging findings and location of the mass. The patient was started on lowdose dexamethasone (4 mg daily), and at the time, orthopedic surgery did not want to biopsy the lesion because of concerns about tumor seeding. The consensus was that tumor debulking could be completed nonemergently.

Tumor staging with computed tomography (CT) of the chest, abdomen, and pelvis with contrast showed no evidence of cortical irregularity or erosive changes of the vertebrae and no additional tumor burden. On day 3 of admission, the patient underwent a CT-guided core biopsy of the epidural mass. MRI of the brain, cervical spine, and thoracic spine revealed no evidence of additional lesions. Dexamethasone was increased to $4 \mathrm{mg}$ every 6 hours because of continued worsening back pain; however, the patient's lower extremity numbness and weakness suddenly worsened. Preliminary microscopy results showed a poorly differentiated malignant neoplasm. Given these results in the setting of a deteriorating neurologic examination, emergent radiation therapy was initiated. The patient received 4 consecutive days of radiation therapy but showed no clinical improvement. Cytology results on admission day 7 were consistent with Ewing sarcoma (Figure 2). That night, the patient underwent urgent lumbar laminectomy with partial facetectomy and foraminotomy of L3-L4 and L4-L5 with extradural tumor resection. Significant functional improvement was noted after the resection, including regaining the ability to ambulate independently with a cane. Steroids were subsequently tapered to $20 \mathrm{mg}$ of prednisone daily for 5 days and then stopped. Whole body positron emission tomography (PET)-CT for staging did not show any evidence of metastasis. Chemotherapy was started on admission day 9 with cyclophosphamide, vincristine, and doxorubicin. The patient was discharged on hospital day 18 to an inpatient rehabilitation facility for 6 days prior to returning home. The patient's care was transferred to a pediatric oncologist to complete chemotherapy with alternating cycles of vincristinedoxorubicin-cyclophosphamide and ifosfamide-etoposide (VDC/IE), with granulocyte colony-stimulating factor (filgrastim) for a total of 6 cycles over 12 weeks, followed by 8 cycles over 16 weeks. PET-CT after cycle 6 was disease free, and CT chest completed at her 14th cycle was without disease. These imaging results were used to determine the patient's remission.

\section{DISCUSSION}

Early recognition of the clinical signs of epidural extraosseous Ewing sarcoma is key to expediting diagnosis and improving prognosis. Most patients with extraosseous Ewing
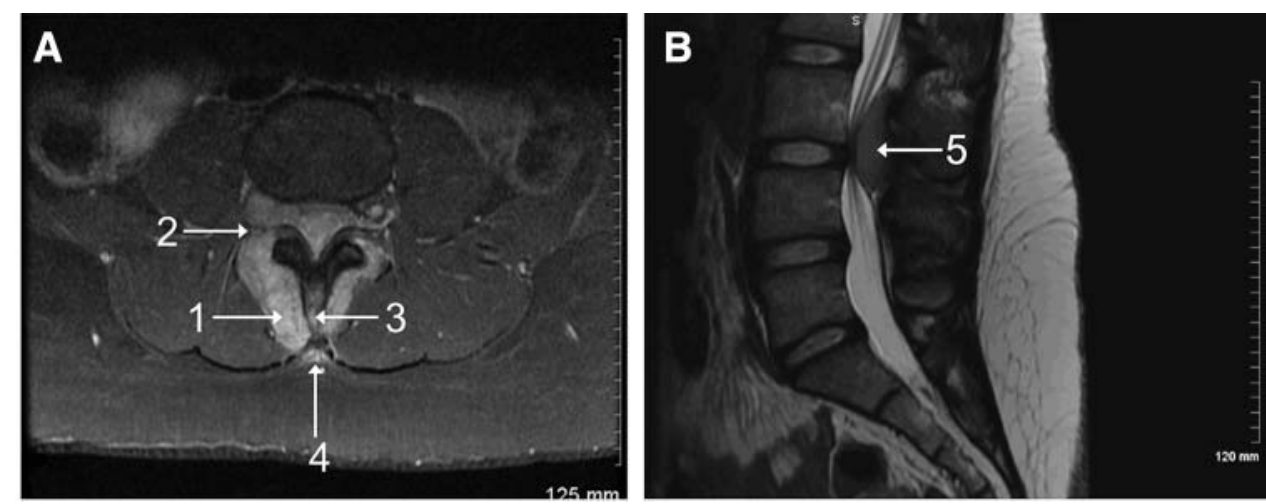

Figure 1. A. Axial magnetic resonance imaging (MRI) of the lumbar spine shows (1) an infiltrative, enhancing dorsal epidural and posterior paraspinal soft tissue mass centered at the L4-L5 level, resulting in (2) right foraminal narrowing. The soft tissue surrounds the posterior elements of L4 and L5 superiorly and L3 at the right inferior facet. Enhancement of the pars interarticularis and superior facets of L4 is minimal, but the enhancement involves the spinous process of L4 and (3) may show some mild cortical destruction. The paraspinal enhancement has (4) subtle infiltration into the paraspinal musculature. B. Sagittal MRI of the lumbar spine demonstrates (5) the same soft-tissue mass causing severe narrowing of the thecal sac at the L4-L5 level. 

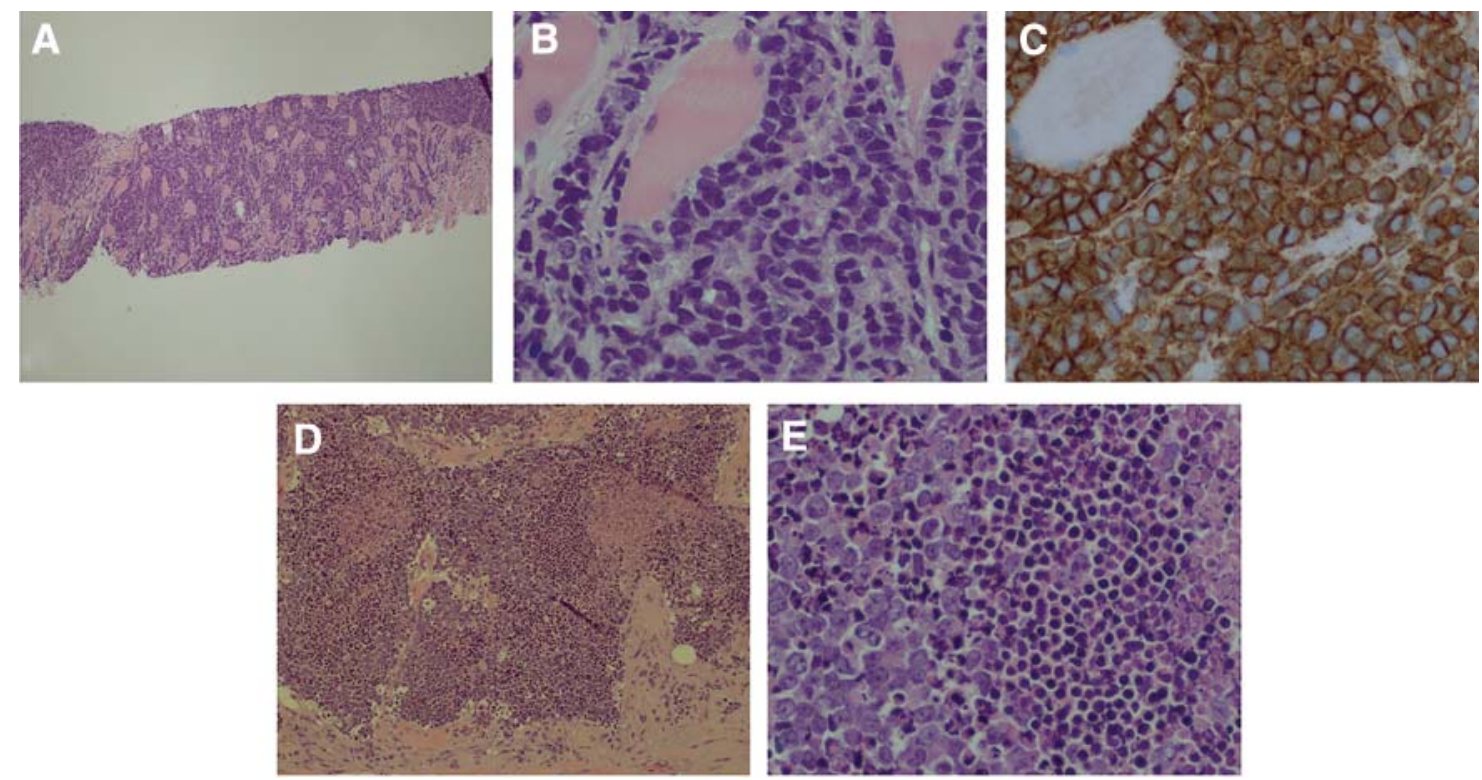

Figure 2. A. Diagnostic core biopsy shows diffuse small round blue cell tumor invading skeletal muscle (hematoxylin and eosin [H\&E] stain, $\times 40$ ). B. Magnified view of diagnostic core biopsy shows small round blue cell tumor invading skeletal muscle (H\&E stain, $\times 400)$. C. Diagnostic core biopsy of the tumor shows strong diffuse CD99 staining (immunohistochemical stain, $\times 400)$. D. Tumor debulking specimen, status post radiation therapy, shows geographic areas of necrosis (H\&E stain, $X$ 40). E. Tumor debulking specimen, status post radiation therapy, shows small round blue cell morphology and significant areas of necrosis (on right) (H\&E stain, $\times 400)$.

sarcoma are diagnosed when the chronic pain acutely worsens after exercise or trauma, similar to the presentation of our case. ${ }^{1}$ In other cases of epidural extraosseous Ewing sarcoma, symptoms have largely been a result of cord compression, including radicular back pain, lower extremity weakness or numbness, and bowel or bladder dysfunction. ${ }^{9}$ While these symptoms are consistently seen in epidural extraosseous Ewing sarcoma, this specific tumor location remains rare. In the more common osseous Ewing sarcoma, bilateral lower extremity weakness is an unusual finding, as only $8 \%$ of osseous Ewing sarcoma involves the spine. ${ }^{1}$ More common symptoms of osseous Ewing sarcoma are pain (82\%-88\% of patients) and a palpable mass (60\% of patients). ${ }^{10}$

To accurately diagnose extraosseous Ewing sarcoma, the clinician must rule out several other potential etiologies of an epidural mass. The most important diagnoses to consider include tumors that histologically mimic the histologic appearance of extraosseous Ewing sarcoma: Iymphoma, embryonal rhabdomyosarcoma, and neuroblastoma. ${ }^{11}$ In our case, lymphoma was the primary consideration, given the epidural location of the mass. Others have reported epidural extraosseous Ewing sarcoma mimicking lymphoma and vice versa. ${ }^{12,13}$ Biopsy with subsequent immunohistochemical staining remains the gold standard for differentiating between these types of tumors.

Even after the histologic diagnosis of Ewing sarcoma was made, this case presented a challenge in determining whether the lesion originated in bone or soft tissue. Although the $\mathrm{MRI}$ showed mild cortical destruction, the bone involvement appeared to be consistent with external erosion secondary to an extraosseous process, a characteristic pattern of extraosseous Ewing sarcoma. ${ }^{3,4,10,14}$ Osseous Ewing sarcoma has a predilection to originate in the medullary canal, a pattern not appreciated on imaging in this case. ${ }^{10}$ Widening of the neural foramen as a result of local spread of the tumor is another feature present in this case that has been commonly reported in cases of epidural extraosseous Ewing sarcoma. ${ }^{11,15}$ More specific diagnostic criteria for extraosseous Ewing sarcoma need to be developed, as accurate diagnosis would allow researchers to distinguish variations in treatment response compared to Ewing sarcoma. Christie et al have proposed such criteria, but they have not yet become widely used. ${ }^{16}$

The most essential component of any workup of an epidural tumor is expediting the time to biopsy. Our case is an excellent example of how differing perspectives from different consultants may hinder this goal. In our case, the recommendation from spinal surgery was to start steroids and observe for symptomatic response. Feeling uneasy about this plan, the primary team contacted neuro-oncology, and those consultants promptly advised a core needle biopsy to identify the tumor. In retrospect, the timely biopsy was crucial to avoid nerve damage and improve prognosis. Rapid diagnosis and treatment are critical in cases of cord compression, as every hour of compression decreases the likelihood of a full neurologic recovery. ${ }^{17}$ Another reason prompt tumor identification is key is the increased risk of metastasis with delayed diagnosis. In the Ewing sarcoma family of tumors, metastasis is the most important prognostic factor. ${ }^{18}$ While overt metastasis is rarely found on presentation of Ewing sarcoma, microscopic metastasis is assumed, as up to $90 \%$ of Ewing sarcoma patients relapse if only treated with local therapy. ${ }^{1}$ This case demonstrates the importance of challenging a consultant opinion when options are limited and prognosis is questionable from a clinical perspective. In this case, seeking a second opinion from a different but 
related discipline resulted in a superior diagnostic and treatment plan.

Consulting a pediatric oncologist at a nearby hospital as soon as the mass was discovered may have placed Ewing sarcoma above lymphoma on the differential. While increased suspicion of Ewing sarcoma may have prevented unnecessary empiric radiation therapy, chemotherapy would still have been held until the pathologic diagnosis was confirmed. Of note, experts on extraosseous Ewing sarcoma throughout the country were consulted immediately after diagnosis to discuss the patient's treatment plan and prognosis.

The treatment of extraosseous Ewing sarcoma has not been sufficiently studied, principally because of the rarity of extraosseous Ewing sarcoma and the absence of consistent guidelines to differentiate Ewing sarcoma from extraosseous Ewing sarcoma. For this reason, the treatment of extraosseous Ewing sarcoma is currently identical to that of Ewing sarcoma and consists of alternating cycles of VDC/IE. ${ }^{19} \mathrm{Ad}-$ junctive radiation therapy and surgery are also often used, depending on the tumor location.

\section{CONCLUSION}

Epidural masses causing cord compression should be biopsied immediately to evaluate for malignancy, including extraosseous Ewing sarcoma. Prognosis can deteriorate with any delay in time to diagnosis, and second opinions should be pursued with vigilant clinical gestalt in the appropriate setting.

\section{ACKNOWLEDGMENTS}

The authors have no financial or proprietary interest in the subject matter of this article.

\section{REFERENCES}

1. Ries LAG, Smith MA, Gurney JG, et al, eds. Cancer Incidence and Survival Among Children and Adolescents: United States SEER Program 1975-1995, National Cancer Institute, SEER Program. NIH Pub. No. 99-4649. Bethesda, MD, 1999. https://seer.cancer .gov/archive/publications/childhood/childhood-monograph .pdf. Accessed September 11, 2018.

2. Sankar S, Lessnick SL. Promiscuous partnerships in Ewing's sarcoma. Cancer Genet. 2011 Jul;204(7):351-365. doi: 10.1016/j. cancergen.2011.07.008.

3. Tefft M, Vawter GF, Mitus A. Paravertebral "round cell" tumors in children. Radiology. 1969 Jun;92(7):1501-1509. doi: 10.1148/ 92.7.1501.

4. Applebaum MA, Worch J, Matthay KK, et al. Clinical features and outcomes in patients with extraskeletal Ewing sarcoma. Cancer. 2011 Jul 1;117(13):3027-3032. doi: 10.1002/cncr.25840.

5. Raney RB, Asmar L, Newton WA Jr, et al. Ewing's sarcoma of soft tissues in childhood: a report from the intergroup rhabdomyosarcoma study, 1972 to 1991. J Clin Oncol. 1997 Feb;15(2):574-582. doi: 10.1200/JCO.1997.15.2.574.
6. Kobayashi S, Takahashi J, Sakashita K, Fukushima M, Kato H. Ewing sarcoma of the thoracic epidural space in a young child. Eur Spine J. 2013 May;22 Suppl 3:373-379. doi: 10.1007/s00586012-2481-y.

7. Bustoros $M$, Thomas $C$, Frenster J, et al. Adult primary spinal epidural extraosseous Ewing's sarcoma: a case report and review of the literature. Case Rep Neurol Med. 2016;2016:1217428. doi: 10.1155/2016/1217428.

8. Scheithauer BW, Egbert BM. Ewing's sarcoma of the spinal epidural space: report of two cases. J Neurology, Neurosurg Psychiatry. 1978 Nov;41(11):1031-1035.

9. Kaspers GJ, Kamphorst W, van de Graaff M, van Alphen HA, Veerman AJ. Primary spinal epidural extraosseous Ewing's sarcoma. Cancer. 1991 Aug 1;68(3):648-654.

10. Murphey MD, Senchak LT, Mambalam PK, Logie Cl, KlassenFischer MK, Kransdorf MJ. From the radiologic pathology archives: Ewing sarcoma family of tumors: radiologicpathologic correlation. Radiographics. 2013 May;33(3):803-831. doi: 10.1148/rg.333135005.

11. Shin JH, Lee HK, Rhim SC, Cho KJ, Choi CG, Suh DC. Spinal epidural extraskeletal Ewing sarcoma: MR findings in two cases. AJNR Am J Neuroradiol. 2001 Apr;22(4):795-798.

12. Ozdemirli M, Fanburg-Smith JC, Hartmann DP, Azumi N, Miettinen M. Differentiating lymphoblastic lymphoma and Ewing's sarcoma: lymphocyte markers and gene rearrangement. Mod Pathol. 2001 Nov;14(11):1175-1182. doi: 10.1038/modpathol.3880455.

13. Lucas DR, Bentley G, Dan ME, Tabaczka P, Poulik JM, Mott MP. Ewing sarcoma vs lymphoblastic lymphoma. A comparative immunohistochemical study. Am J Clin Pathol. 2001 Jan;115 (1):11-17. doi: 10.1309/K1XJ-6CXR-BQQU-V255.

14. Albarello F, Deriu G, Goletti S, Campioni P. Extraosseus Ewing sarcoma: an uncommon periclavicular location. Radiol Case Rep. 2016 Feb 17;10(2):1117. doi: 10.2484/rcr.v10i2.1117.

15. Kennedy JG, Eustace S, Caulfield R, Fennelly DJ, Hurson B, O'Rourke KS. Extraskeletal Ewing's sarcoma: a case report and review of the literature. Spine (Phila Pa 1976). 2000 Aug 1;25 (15):1996-1999.

16. Christie DR, Bilous AM, Carr PJ. Diagnostic difficulties in extraosseous Ewing's sarcoma: a proposal for diagnostic criteria. Australas Radiol. 1997 Feb 4;41(1):22-28.

17. Putz C, van Middendorp JJ, Pouw MH, et al. Malignant cord compression: a critical appraisal of prognostic factors predicting functional outcome after surgical treatment. $J$ Craniovertebr Junction Spine. 2010 Jul;1(2):67-73. doi: 10.4103/ 0974-8237.77670.

18. Leavey PJ, Mascarenhas L, Marina N, et al. Prognostic factors for patients with Ewing sarcoma (EWS) at first recurrence following multi-modality therapy: a report from the Children's Oncology Group. Pediatr Blood Cancer. 2008 Sep;51(3):334-338. doi: 10.1002/pbc.21618.

19. Galyfos G, Karantzikos GA, Kavouras N, Sianou A, Palogos K, Filis K. Extraosseous Ewing sarcoma: diagnosis, prognosis and optimal management. Indian J Surg. 2016 Feb;78(1):49-53. doi: 10.1007/s12262-015-1399-0.

This article meets the Accreditation Council for Graduate Medical Education and the American Board of Medical Specialties Maintenance of Certification competencies for Patient Care and Medical Knowledge. 